African Crop Science Journal by African Crop Science Society is licensed under a Creative Commons Attribution 3.0 Uganda License. Based on a work at www.ajol.info/ and www.bioline.org.br/cs DOI: https://dx.doi.org/10.4314/acsj.v26i4.3

\title{
OVERLAPPING QUANTITATIVE TRAIT LOCI INDICATION OF SIMILARITY OF RICE RESPONSE TO VARIOUS STRESSES
}

\author{
A.O. CHARLES JOSEPH \\ University of Dar es Salaam, Department of Botany, P. O. Box 35060, Dar es Salaam, Tanzania \\ Corresponding author: jcharles@udsm.ac.tz
}

(Received 14 April, 2018; accepted 12 November, 2018)

\begin{abstract}
Combing stresses tolerance and grain yield is key for rice breeding to cope with the effect of climate change. The objective of this study was identify; lines resistant to multiple stresses and chromosomal segments containing QTLs conferring tolerance to both abiotic and biotic stresses Results revealed that, ratio of trait value under stress to normal conditions indicated that grain yield (GY) was the most affected trait by the stresses while grain size related traits were the least affected. Within the 29 regions harboring 50 overlapping QTLs, 39 QTLs were detected under drought, cold and bacterial blight stresses and 11 QTLs were stress specific. Overlapping regions harboring QTLs controlling multiples traits in different genetic backgrounds and in four environments (drought, cold, bacterial blight and normal condition (DCBN) were detected. Several lines offering resistance to both abiotic and biotic stresses were also observed. The study indicated that to some degree, plants respond to various stresses in a similar way. It also indicated that drought, BB and low temperature tolerance can be improved simulteniously, while retaining economical yield under stress; and at the same time higher yield during favorable conditions.
\end{abstract}

Key Words: Bacterial blight, cold stress, Oryza sativa

\section{RÉSUMÉ}

La combinaison de la tolérance aux stress et le rendement en grains est une clé pour l'amélioration génétique du riz contre les effets des changements climatiques. L'objective de cette étude a été d'identifier ; les lignées résistantes aux stress multiples et les segments de chromosomes contenant les QTLs conférant la tolérance aux stress abiotiques and biotiques. Les résultats ont révélé que, le ratio de la valeur des traits sous stress et dans les conditions normales a montré que le rendement en grain $(\mathrm{GY})$ a été le trait le plus affecté par les stress alors que les traits relatifs à la taille du grain ont été les moins affectés. Dans les 29 régions comportant les 50 QTLs redondants, 39 QTLs ont été détectés sous le stress de la sécheresse, du froid et du mildiou bactérien et 11 QTLs ont été spécifiques aux stress. Les régions de redondance contenant les QTLs contrôlant les traits multiples dans des origines génétiques différentes et dans quatre environnements (sécheresse, froid, mildiou bactérien et conditions normales $(\mathrm{DCBN}))$ ont été détectées. Plusieurs lignées offrant de résistance à la fois aux stress biotiques et abiotiques ont été observées. L'étude a indiqué qu'à certain degré, les plantes répondent aux différents stress de manière similaire. Elle a aussi indiqué que la sécheresse, la tolérance au BB et à la faible température peut être améliorée simultanément, de même que maintenir le rendement économique sous stress ; et un rendement élevé dans des conditions favorables.

Mots Clés: Mildiou bactérien, stress du froid, Oryza sativa 


\section{INTRODUCTION}

Rice (Oryza sativa L.) is a major food crop for majority of people in developing countries. Due to increasing effects of abiotic stresses (such as drought, submergence, salinity, heavy metal contamination and nutrient deficiency), biotic stresses (such as bacteria, viruses, fungi, or nematodes affect), scarce natural resources and perhaps narrow genetic pools of elite lines, increasing rice productivity to meet global energy demand is a challenge. The fact that stresses co-exist under certain natural environments, further makes it harder to improve rice yield. For instance, drought and low temperature in the northern hemisphere (Zhang et al., 2012), cold-dry temperatures in the highland plains, and higher relative humidity and cloudy nights in the coastal regions co-exist. But also, during the course of drought stress development, pathogens can infect plants or drought stress can occur on already pathogen infected plants. Many studies have shown that the cellular responses to these environmental challenges are rather similar (Peng et al., 2011; Cao et al., 2011; Xu et al., 2011; Wang et al., 2012; Zhang et al., 2012; Sharma et al., 2013; Shaik and Ramakrishna, 2014).

Pathogen infection on already drought stressed plants enhances plant resistance to pathogen through drought-induced activation of basal defense (such as enhanced induction of antimicrobial and PR-proteins activation) or increased susceptibility due to weakened basal defense (such as high levels of ABA in drought stressed plants, which interfere with pathogen-induced plant defense signaling and thereby reduce the expression of defenserelated genes). On the other hand, exposure of pathogen infected plants to drought stress increases drought stress tolerance of plants through pathogen-induced salicylic acid (SA)dependent ROS signaling, or result in susceptibility of the plants to drought stress due to SA or jasmonic acid (JA)-mediated reduction in responsiveness of plants to abscisic acid (ABA) (Ramegowda and SenthilKumar, 2015).

Exposure of plants to drought stress and pathogen infection simultaneous can also result in tolerance to both stresses due to inherent ability of plants to induce unique tailored strategies; or can make plants more susceptible to both stresses due to weakened fitness of plants due to exacerbation of damage caused by one stress on other (Ramegowda and Senthil-Kumar, 2015). Combined effects under both abiotic and biotic stress responses have been illustrated using APETALA2 (AP2) (Xu et al., 2011), lesion-simulating disease (Epple et al., 2003) and abscisic acid-induced myb1 (SlAIM1) gene (Abuqamar et al., 2009). Overlapping QTLs (OQs) between lowtemperature and drought in soybeans (Zhang et al., 2012); salt and low temperature in soybeans (Peng et al., 2011), drought and sheath blast in rice, (Zheng et al., 2007) and salt and drought in rice (Xu et al., 2011, Wang et al., 2012) have been reported. However, genetic interactions between low temperate, bacterial blight and drought simultaneously have not been studied. Thus, the objective of this study was to identify tolerant lines to low temperature, BB and drought stresses and the corresponding loci coffering resistances to theses stresses were identified.

\section{METHODOLOGY}

Two sets of reciprocal inbred lines, consisting of 226 lines $\left(\mathrm{BC}_{2} \mathrm{~F}_{8}\right)$ with the MH63 background (MH63_ILs), 229 lines $\left(\mathrm{BC}_{2} \mathrm{~F}_{8}\right)$ with the 02428 background (02428_IL) and 262 (F2:8) recombinant inbreed lines (RILs), developed from a across between Minghui63 (MH63) indica and 02428 japonica were used in this study. Three BB inoculums (C5, V5 and P6) were prepared according to (Ou, 1985). Field experiments conducted at Hainan-China were arranged in a completely randomised block design, with two replications.

In each plot, 10 plants were planted in a row with the spacing of $20 \mathrm{~cm} \times 25 \mathrm{~cm}$. 
Inoculation was done at vegetative stage, by cutting off the flag leaf and beneath leaves tip using scissors previously dipped in bacterial suspension (Kauffman et al., 1973). After 21 days, both lesion length and leaf total length were measured using a ruler. Lesion length of $>0-5 \mathrm{~cm}$ long was regarded as resistant $(\mathrm{R})$, $>5-10 \mathrm{~cm}$ moderately resistant (MR), $>10$ $15 \mathrm{~cm}$ moderately susceptible (MS), $>15 \mathrm{~cm}$ susceptible (S). Diseased leaf area was calculated as:

$\frac{\text { Lesion length }}{\text { Total leaf length }} \times 100$

Where:

$<5 \%$ was regarded as resistant (R) $6-12 \%$ moderate resistant, $13-25 \%$ moderate susceptible and $>25 \%$ susceptible. In drought treatment, approximately after 60 days of transplanting, fields were drained. Field was maintained dry until panicles of all plants excerted completely. Then, re-irrigation was resumed only once until the harvesting. In the normal irrigation (control), irrigation was applied whenever necessary until lines reached the grain filling stage. Cold treatment was initiated by growing rice at three different locations of high altitude at Yunnan province at temperature less than $15^{\circ} \mathrm{C}$.

Heading date was recorded when $50 \%$ of the plants within a row flowered. At maturity, plant height was measured using a ruler and effective tiller number for both infected and uninfected plants were recorded. Flag leaf length, flag leaf width and flag leaf rolling were measured for the unaffected plants.

During harvesting, four panicles of the main tiller and four hills for BB infected and BB uninfected plants for each race were harvested separately. Harvested panicles and hills were sun dried; then panicle length, spikelet number per panicle, filled grains numbers per panicle, spikelet fertility, 1000-grain weight, yield per plant, grain length, grain width and grain length width ratio were evaluated separately.
For genotyping, 265 high-quality SNPs were used for a consensus linkage map construction. GLM proc SAS version 9.2 (SAS institute, 2002). For SNP genotyping, Minghui63 (MH63) and 02428, were submitted to whole genome re-sequencing and a total of 265 high-quality SNP evenly design by Illumnia Corp, USA and genotyping assay by Xing-Wang Deng's Lab in Peking University. For Linkage map construction and QTL mapping, a total of 265 high-quality SNP markers developed based on sequencing and evenly distributed throughout the 12 chromosomes with an average distance of 4.27 $\mathrm{cM}$ between adjacent markers, were used for genotyping assay. The total length of the consensus map was $1132.9 \mathrm{cM}$. Additive and epistasis QTLs for yield and yield component traits were identified by the method of Inclusive Composite Interval Mapping (ICIM) and Inclusive Composite Interval Mapping of digenic epistatic (ICIM-EPI) in Icimapping ver. 4.0 presented in centi-morgans (cM), using the Kosambi function (Wang et al., 2016). The thresholds of LOD 2.5 for declaring additive QTL and 5 for Epistasis QTL were used. GGT2 (Ralph, 2007) for trait marker association and estimating proportion of recurrent genome in the selected individuals were used and Genetic map was drawn using Liu and Meng (2003) MapDraw.

\section{RESULTS}

ILs and parents performance. The yield related trait performances for introgression lines and their parents under drought stress, bacterial blight stress (BB) and low temperature; and normal irrigations are presented in Table 1. MH63 (P2) had longer heading period than 02428 (P1) under drought stress, BB stress and under low temperature in all seasons and locations. P2 also had more panicles in both drought and BB stresses, but very few panicle numbers, total grain number per panicle, filled grain number, unfilled grain number and spikelet fertility under low temperatures, indicating that $\mathrm{P} 1$ was more cold 
TABLE 1. Performance of parents and BILs for BB, drought and low temperature tolerance related traits in rice

\begin{tabular}{|c|c|c|c|c|c|c|c|c|}
\hline \multirow[t]{2}{*}{ Environment } & \multirow[t]{2}{*}{ Trait } & \multicolumn{3}{|c|}{ Parents } & \multicolumn{2}{|c|}{ MH63_ILs } & \multicolumn{2}{|c|}{ 02428_ILs } \\
\hline & & P1 & $\mathrm{P} 2$ & P1-P2 & $\mathrm{M} \pm \mathrm{SD}$ & Range & $\mathrm{M} \pm \mathrm{SD}$ & Range \\
\hline \multirow[t]{15}{*}{ DS 2013} & HD & 100.3 & 121.0 & -20.7 & $115.8 \pm 8.0$ & $97-127$ & $106.0 \pm 10$ & $87-127$ \\
\hline & $\mathrm{PH}$ & 59.8 & 65.8 & -6.0 & $65.4 \pm 7.5$ & $50-90$ & $61.2 \pm 8.1$ & $21.8-88$ \\
\hline & PL & 17.9 & 19.4 & -1.5 & $19.0 \pm 2.1$ & $14.1-26$ & $17.1 \pm 1.8$ & $13-21.5$ \\
\hline & TN & 5.0 & 7.0 & -2.0 & $6.7 \pm 1.3$ & $4-10.0$ & $4.6 \pm 1.2$ & $0.8-8.4$ \\
\hline & SNP & 106.7 & 72.3 & 34.4 & $78.1 \pm 31.7$ & $42-216.0$ & $92.5 \pm 24.7$ & $44-205.2$ \\
\hline & FGN & 80.0 & 41.7 & 38.3 & $53.6 \pm 28.8$ & $19-142.0$ & $63.5 \pm 25.3$ & $2-162.8$ \\
\hline & $\mathrm{SF}$ & 71.6 & 58.0 & 13.6 & $66.5 \pm 12.2$ & $31-92.0$ & $67.6 \pm 17.5$ & $3.4-90.9$ \\
\hline & TGW & 18.7 & 24.4 & -5.7 & $24.1 \pm 4.7$ & $10.45-33.8$ & $19.7 \pm 4.9$ & $0.8-33.1$ \\
\hline & GY & 5.7 & 7.4 & -1.7 & $7.3 \pm 2.8$ & $3.13-17.5$ & $5.0 \pm 1.9$ & $1.8-12.2$ \\
\hline & GL & 6.9 & 9.7 & -2.8 & $9.4 \pm 0.5$ & $7.74-10.3$ & $7.2 \pm 0.6$ & $6.0-9.2$ \\
\hline & GW & 3.4 & 2.6 & 0.8 & $2.7 \pm 0.2$ & $2.43-3.1$ & $3.3 \pm 0.3$ & $2.1-3.7$ \\
\hline & GLW & 2.0 & 3.7 & -1.7 & $3.5 \pm 0.3$ & $2.8-4.0$ & $2.2 \pm 0.3$ & $1.8-4.0$ \\
\hline & GT & 2.2 & 2.0 & 0.2 & $2.0 \pm 0.1$ & $1.7-2.2$ & $2.1 \pm 01$ & $1.4-2.5$ \\
\hline & GLT & 3.2 & 5.0 & -1.8 & $4.8 \pm 0.4$ & $3.7-5.9$ & $3.4 \pm 0.4$ & $2.6-5.3$ \\
\hline & GWT & 1.6 & 1.3 & 0.2 & $1.4 \pm 0.1$ & $1.1-1.7$ & $1.5 \pm 0.1$ & $1.2-2.3$ \\
\hline \multirow[t]{15}{*}{ DS 2014} & HD & 108.2 & 129.8 & -21.7 & $122.5 \pm 8.6$ & 97-137 & $108.2 \pm 4.0$ & $100-117$ \\
\hline & $\mathrm{PH}$ & 66.1 & 67.0 & -0.9 & $67.2 \pm 7.3$ & $49.7-97.4$ & $65.1 \pm 8.4$ & $43-81.8$ \\
\hline & PL & 19.8 & 18.5 & 1.3 & $19.4 \pm 2.1$ & $14.9-26.7$ & $19.3 \pm 1.7$ & $14.7-23.2$ \\
\hline & $\mathrm{TN}$ & 9.6 & 10.0 & -0.4 & $9.2 \pm 2.1$ & 3.1-16 & $7.2 \pm 1.7$ & $3.1-14.2$ \\
\hline & SNP & 135.5 & 94.3 & 41.1 & $107.3 \pm 37.6$ & $52.8-217$ & $129.7 \pm 32.2$ & $58-227.6$ \\
\hline & FGN & 122.1 & 73.6 & 48.5 & $74.3 \pm 32.4$ & 25-117 & $103.6 \pm 37.3$ & 17-205 \\
\hline & $\mathrm{SF}$ & 90.1 & 77.4 & 12.7 & $68.3 \pm 10.5$ & $41.8-87.3$ & $78.5 \pm 15.7$ & $19.8-98.1$ \\
\hline & TGW & 17.9 & 22.2 & -4.2 & $22.2 \pm 3.6$ & 6.4-29.1 & $18.9 \pm 3.1$ & $11.6-26.3$ \\
\hline & GY & 10.8 & 6.0 & 4.7 & $6.5 \pm 4.9$ & $0.7-20.0$ & $8.2 \pm 4.8$ & $0.5-23.3$ \\
\hline & GL & 6.4 & 9.0 & -2.6 & $8.8 \pm 0.5$ & 7.4-9.7 & $7.0 \pm 0.6$ & 6.1-8.7 \\
\hline & GW & 3.4 & 2.7 & 0.7 & $2.8 \pm 0.1$ & $2.3-3.3$ & $3.2 \pm 0.2$ & $2.4-3.7$ \\
\hline & GLWR & 1.9 & 3.5 & -1.5 & $3.2 \pm 0.2$ & $2.7-3.8$ & $2.2 \pm 0.3$ & $1.8-3.6$ \\
\hline & FLL & 22.4 & 17.1 & 5.3 & $19.6 \pm 3.6$ & $13.4-35.7$ & $22.9 \pm 3.6$ & $14.2-43.8$ \\
\hline & FLW & 1.7 & 1.9 & -0.3 & $1.8 \pm 0.2$ & 1.5-2.2 & $1.7 \pm 0.2$ & $1.1-2.1$ \\
\hline & FLR & 1.6 & 1.7 & -0.2 & $1.7 \pm 0.2$ & $1.1-2.2$ & $1.5 \pm 0.2$ & $0.8-2.8$ \\
\hline \multirow[t]{16}{*}{ BB stress } & C5 & 7.0 & 3.5 & 3.5 & $3.4 \pm 1.7$ & $0.5-15.1$ & $7.7 \pm 3.2$ & $0.5-18.6$ \\
\hline & FLL & 30.5 & 27.3 & 3.2 & $26.3 \pm 3.4$ & $17.1-39.3$ & $28.9 \pm 4.4$ & $17.2-58.1$ \\
\hline & FLW & 2.0 & 1.9 & 0.1 & $1.8 \pm 0.2$ & 1.3-3.1 & $1.9 \pm 1.1$ & $1.1-22.1$ \\
\hline & FLR & 1.8 & 1.8 & 0.0 & $1.7 \pm 0.2$ & 1.3-4.7 & $1.6 \pm 0.3$ & $0.7-5.1$ \\
\hline & $\mathrm{PH}$ & 83.8 & 90.5 & -6.7 & $89.5 \pm 5.2$ & $70.7-112.3$ & $85.0 \pm 7.6$ & $57-124$ \\
\hline & $\mathrm{TN}$ & 10.8 & 11.2 & -0.4 & $10.4 \pm 2.2$ & 5.5-30.6 & $9.5 \pm 2.3$ & $5.2-25$ \\
\hline & HD & 109.6 & 121.3 & -11.7 & $119.2 \pm 3.5$ & 108-129 & $109.1 \pm 13.9$ & 96-141 \\
\hline & GY & 85.2 & 97.1 & -11.9 & $84.3 \pm 23.8$ & $14.5-155.4$ & $69.6 \pm 23.6$ & $9.7-153.7$ \\
\hline & PL & 21.4 & 24.2 & -2.8 & $23.2 \pm 1.7$ & $16.2-27.4$ & $21.0 \pm 1.9$ & $13.6-26.9$ \\
\hline & SNP & 624.3 & 539.2 & 85.1 & $544 \pm 120.1$ & 286-1117 & $688.9 \pm 166.3$ & 251-1337 \\
\hline & FGN & 561.3 & 450.0 & 111.3 & $435.2 \pm 106.4$ & 207-901 & $560.4 \pm 150.9$ & $169-1066$ \\
\hline & $\mathrm{SF}$ & 90.0 & 82.9 & 7.1 & $80.1 \pm 9.2$ & $46.9-95.6$ & $81.6 \pm 12.0$ & $25.8-98.2$ \\
\hline & TGW & 22.2 & 27.4 & -5.1 & $27.0 \pm 2.7$ & $16.2-34.3$ & $22.8 \pm 3.1$ & 8.6-32.3 \\
\hline & GL & 7.4 & 9.5 & -2.1 & $9.5 \pm 0.5$ & $6.7-10.7$ & $7.4 \pm 0.8$ & $6.1-9.9$ \\
\hline & GW & 3.5 & 2.8 & 0.6 & $2.9 \pm 0.1$ & 2.6-3.5 & $3.5 \pm 0.3$ & $2.3-3.9$ \\
\hline & GLW & 2.2 & 3.4 & -1.2 & $3.3 \pm 0.2$ & $1.9-4$ & $2.1 \pm 0.4$ & $1.7-3.7$ \\
\hline
\end{tabular}


TABLE 1. Contd.

\begin{tabular}{|c|c|c|c|c|c|c|c|c|}
\hline \multirow[t]{2}{*}{ Environment } & \multirow[t]{2}{*}{ Trait } & \multicolumn{3}{|c|}{ Parents } & \multicolumn{2}{|c|}{ MH63_ILs } & \multicolumn{2}{|c|}{ 02428_ILs } \\
\hline & & $\mathrm{P} 1$ & $\mathrm{P} 2$ & $\mathrm{P} 1-\mathrm{P} 2$ & $\mathrm{M} \pm \mathrm{SD}$ & Range & $\mathrm{M} \pm \mathrm{SD}$ & Range \\
\hline \multirow[t]{6}{*}{ LT SongMing (2014) } & HD & 142.0 & 155.0 & -13.0 & $133.9 \pm 7.1$ & $110-163$ & $137.5 \pm 5.2$ & $124.5-153$ \\
\hline & $\mathrm{PN}$ & 5.0 & 4.0 & 1.0 & $4.8 \pm 0.6$ & $2-5$ & $4.6 \pm 0.5$ & 3-5.5 \\
\hline & FGN & 402.3 & 514.0 & -111.7 & $477.5 \pm 219$ & 2-1040 & $435.8 \pm 226$ & 3-919 \\
\hline & UGN & 731.7 & 324.0 & 407.7 & $552.4 \pm 239$ & $129-1616$ & $579.6 \pm 214$ & $170.5-1296$ \\
\hline & SNP & 1134.0 & 838.0 & 296.0 & $1029.9 \pm 244$ & $299-1760$ & $1015.3 \pm 266$ & 250-1590 \\
\hline & $\mathrm{SF}$ & 35.6 & 61.3 & -25.7 & $46.3 \pm 18.4$ & $0.22-82.9$ & $41.8 \pm 17$ & $0.24-84.4$ \\
\hline \multirow[t]{6}{*}{ LT Xudian (2014) } & HD & 133.1 & 133.3 & -0.3 & $135.10 \pm 5.10$ & $115-149$ & $136.4+5.8$ & $115-154$ \\
\hline & $\mathrm{PN}$ & 5.1 & 4.8 & 0.3 & $4.815 \pm 0.45$ & $3.5-5.5$ & $4.7+0.5$ & 3-5.5 \\
\hline & FGN & 466.0 & 340.6 & 125.4 & $500.46 \pm 200$ & $28-849.5$ & $442.5+235$ & $12-998$ \\
\hline & UGN & 338.8 & 434.0 & -95.2 & $329.11 \pm 129$ & $70.5-739.5$ & $381.58+210$ & $79.5-1447$ \\
\hline & SNP & 804.7 & 774.5 & 30.2 & $829.57 \pm 158$ & $378-1096$ & $824.1+219$ & $252.5-1565$ \\
\hline & $\mathrm{SF}$ & 54.1 & 40.6 & 13.5 & $57.94 \pm 19.0$ & $4.93-89.52$ & $52.2+22$ & 1.4-88.1 \\
\hline
\end{tabular}

$\mathrm{HD}=$ days to flowering; $\mathrm{PH}=$ plant height $; \mathrm{PL}=$ panicle length $; \mathrm{PN}=$ panicle number $; \mathrm{GNP}=$ spikelet number per panicle; FGN = filled grain number; $\mathrm{SF}=$ spikelet fertility; TGW = thousand grain weight; $\mathrm{GY}=$ grain weight per plant; GL = grain length; GW = grain width; GLW = grain length width ratio. $\mathrm{UGN}=$ unfiled grain number. DS $=$ drought stress, $\mathrm{BB}=$ bacterial blight, $\mathrm{CT}=$ Low temperature, P1;02428_IL and P2; MH63

tolerant than P2. The performance of BILs skewed toward the recurrent parents, but with transgressive lines in all stresses.

The ratio of trait values under drought stress to normal condition and BB to control conditions are presented in Table 2. Under drought stress, grain size components, especially grain length, grain width, grain thickness, grain length width ratio, grain length thickness ratio and grain width thickness ratio were least affected by drought stress; while grain yield and filled grain number were the most affected.

Drought tolerance versus BB resistance. Correlations between drought tolerance and $\mathrm{BB}$ resistant related traits are presented on Table 3. Except for spikelet fertility in MH63_IL population, all traits showed a positive correlations with each other. For example, the correlation coefficients of GL, GW and GLW between DS and BB was 0.64, 0.54 and 0.61 in MH64_IL background and 0.84, 0.83 and 0.90 in 02428_IL backgrounds. GNP and FGN were moderately affected were $r$, were 0.50 and 0.44 in MH63_IL and 0.48, 0.45 in
02428_IL; while GY had least correlation whereby $\mathrm{r}$ was 0.27 in both populations. Degrees of association differed on trait and genetic background, and stress type. For example, associations between HD with PL, SNP, FGN and GY under stress conditions were $0.34,0.44,0.43$ and 0.42 for BB and 0.08 , $0.28,0.15$ and 0.25 in 02428 IL for drought resistant; but only $(0.2,0.26,0.18$ and 0.09 in MH63_IL and 0.34,0.002,0.02 and 0.17 in 02428_IL under normal conditions.

Introgression patterns of multiple stress tolerant lines. Tolerant lines to multiple stresses and their introgression patterns are presented in Table 3 and their subsequent introgression patterns in Figure 2. In total, 11 lines were tolerant to both Bacterial blight stress and low-temperature stress (BBCT); 6 lines were tolerant to both bacterial blight and drought stress (BBDT), 2 lines tolerant to low temperature and drought stress (CTDT) and four lines tolerant to all stresses (BBDTCT) .

Graphical genotype data of the chromosomes (Figs.1 and 2) of the tolerance lines shows that most overlapping regions 
TABLE 2. Ratios of yield related trait performance under drought stress (DS) and bacterial stress (BB) to control conditions (CTRL) in rice

\begin{tabular}{|c|c|c|c|c|c|c|c|c|}
\hline \multirow[t]{2}{*}{ Ratio } & \multirow[t]{2}{*}{ Traits } & \multicolumn{3}{|c|}{ Parents } & \multicolumn{2}{|c|}{ MH63_ILs } & \multicolumn{2}{|c|}{ 02428_ILs } \\
\hline & & $\mathrm{P} 1$ & P2 & P1-P2 & $\mathrm{M} \pm \mathrm{SD}$ & Range & $\mathrm{M} \pm \mathrm{SD}$ & Range \\
\hline \multirow[t]{15}{*}{ DS/ CTRL (2013) } & HD & 0.97 & 1.11 & -0.14 & $1.09 \pm 0.03$ & $1.02-1.19$ & $1.04 \pm 0.07$ & $0.73-1.16$ \\
\hline & PH & 0.69 & 0.78 & -0.08 & $0.78 \pm 0.06$ & $0.69-0.99$ & $0.70 \pm 0.05$ & $0.52-0.81$ \\
\hline & PL & 0.83 & 0.84 & -0.01 & $0.88 \pm 0.08$ & $0.75-1.26$ & $0.80 \pm 0.07$ & $0.63-0.91$ \\
\hline & $\mathrm{TN}$ & 0.53 & 0.77 & -0.24 & $0.88 \pm 0.17$ & $0.50-1.42$ & $0.53 \pm 0.12$ & $0.18-0.89$ \\
\hline & SNP & 0.61 & 0.56 & 0.05 & $0.64 \pm 0.21$ & $0.36-1.83$ & $0.51 \pm 0.08$ & $0.37-0.93$ \\
\hline & FGN & 0.51 & 0.42 & 0.09 & $0.56 \pm 0.20$ & $0.29-1.42$ & $0.42 \pm 0.11$ & $0.12-0.88$ \\
\hline & SF & 0.79 & 0.75 & 0.03 & $0.86 \pm 0.16$ & $0.52-1.48$ & $0.82 \pm 0.19$ & $0.28-1.74$ \\
\hline & TGW & 0.84 & 0.84 & 0.00 & $0.81 \pm 0.10$ & $0.52-0.98$ & $0.82 \pm 0.18$ & $0.12-1.04$ \\
\hline & GY & 0.31 & 0.33 & -0.02 & $0.40 \pm 0.14$ & $0.18-0.80$ & $0.25 \pm 0.06$ & $0.11-0.36$ \\
\hline & GL & 1.02 & 1.00 & 0.02 & $0.98 \pm 0.03$ & $0.88-1.03$ & $1.00 \pm 0.05$ & $0.89-1.24$ \\
\hline & GW & 0.98 & 0.96 & 0.02 & $0.95 \pm 0.05$ & $0.79-1.05$ & $0.97 \pm 0.05$ & $0.82-1.24$ \\
\hline & GLW & 1.04 & 1.04 & 0.00 & $1.03 \pm 0.06$ & $0.87-1.26$ & $1.02 \pm 0.08$ & $0.72-1.51$ \\
\hline & GT & 1.03 & 1.00 & 0.03 & $0.99 \pm 0.04$ & $0.87-1.08$ & $1.01 \pm 0.05$ & $0.86-1.13$ \\
\hline & GLT & 0.99 & 1.00 & -0.01 & $0.10 \pm 0.05$ & $0.86-1.14$ & $0.98 \pm 0.07$ & $0.80-1.38$ \\
\hline & GWT & 0.95 & 0.96 & -0.01 & $0.97 \pm 0.06$ & $0.80-1.12$ & $0.96 \pm 0.05$ & $0.89-1.15$ \\
\hline \multirow[t]{12}{*}{ DS / CTRL (2014) } & HD & 1.00 & 1.07 & -0.07 & $1.04 \pm 0.05$ & $0.83-1.12$ & $1.00 \pm 0.02$ & $0.90-1.10$ \\
\hline & $\mathrm{PH}$ & 0.80 & 0.70 & 0.10 & $0.73 \pm 0.04$ & $0.63-0.82$ & $0.75 \pm 0.07$ & $0.55-0.87$ \\
\hline & PL & 0.89 & 0.75 & 0.14 & $0.84 \pm 0.10$ & $0.70 \pm 1.11$ & $0.87 \pm 0.07$ & $0.69-1.01$ \\
\hline & $\mathrm{TN}$ & 0.92 & 0.93 & -0.01 & $0.86 \pm 0.15$ & $0.47 \pm 1.17$ & $0.74 \pm 0.14$ & $0.47-1.20$ \\
\hline & SNP & 0.72 & 0.72 & 0.00 & $0.73 \pm 0.17$ & $0.48 \pm 1.23$ & $0.68 \pm 0.13$ & $0.43-1.01$ \\
\hline & FGN & 0.74 & 0.63 & 0.11 & $0.62 \pm 0.21$ & $0.33 \pm 1.26$ & $0.65 \pm 0.16$ & $0.19-1.02$ \\
\hline & $\mathrm{SF}$ & 1.02 & 0.87 & 0.15 & $0.84 \pm 0.12$ & $0.62 \pm 1.13$ & $0.95 \pm 0.15$ & $0.40-1.35$ \\
\hline & TGW & 0.80 & 0.77 & 0.03 & $0.82 \pm 0.11$ & $0.24 \pm 1.05$ & $0.76 \pm 0.09$ & $0.55-1.04$ \\
\hline & GY & 0.58 & 0.24 & 0.35 & $0.28 \pm 0.17$ & $0.05 \pm 0.89$ & $0.46 \pm 0.19$ & $0.06-1.21$ \\
\hline & GL & 0.99 & 0.92 & 0.07 & $0.94 \pm 0.03$ & $0.87 \pm 1.04$ & $0.99 \pm 0.03$ & 0.84-1.09 \\
\hline & GW & 0.96 & 0.95 & 0.00 & $0.96 \pm 0.05$ & $0.84 \pm 1.07$ & $0.95 \pm 0.03$ & $0.84-1.03$ \\
\hline & GLW & 1.04 & 0.98 & 0.06 & $0.97 \pm 0.05$ & $0.88 \pm 1.08$ & $1.04 \pm 0.05$ & $0.88-1.32$ \\
\hline
\end{tabular}


TABLE 2. Contd.

\begin{tabular}{|c|c|c|c|c|c|c|c|c|}
\hline \multirow[t]{2}{*}{ Ratio } & \multirow[t]{2}{*}{ Traits } & \multicolumn{3}{|c|}{ Parents } & \multicolumn{2}{|c|}{ MH63_ILs } & \multicolumn{2}{|c|}{02428 _ILs } \\
\hline & & $\mathrm{P} 1$ & $\mathrm{P} 2$ & P1-P2 & $\mathrm{M} \pm \mathrm{SD}$ & Range & $\mathrm{M} \pm \mathrm{SD}$ & Range \\
\hline & FLL & 0.74 & 0.63 & 0.11 & $0.73 \pm 0.12$ & $0.58 \pm 1.14$ & $0.76 \pm 0.07$ & $0.66-1.08$ \\
\hline & FLW & 0.87 & 0.97 & -0.10 & $0.94 \pm 0.04$ & $0.85 \pm 1.05$ & $0.88 \pm 0.07$ & $0.65-1.01$ \\
\hline & FLR & 0.98 & 0.94 & 0.04 & $0.92 \pm 0.05$ & $0.81 \pm 1.03$ & $0.95 \pm 0.12$ & $0.69-1.46$ \\
\hline \multirow[t]{11}{*}{ BB / CTRL (2014) } & $\mathrm{PH}$ & 0.97 & 0.98 & -0.02 & $1.00 \pm 0.06$ & $0.37-1.39$ & $0.99 \pm 04$ & $0.81-1.31$ \\
\hline & $\mathrm{TN}$ & 0.95 & 1.23 & -0.28 & $1.03 \pm 0.21$ & $0.43-1.77$ & $1.09 \pm 0.21$ & $0.46-1.95$ \\
\hline & GY & 1.02 & 1.09 & -0.06 & $1.01 \pm 0.30$ & $0.28-1.95$ & $1.11 \pm 0.29$ & $0.29-2.00$ \\
\hline & PL & 1.00 & 0.99 & 0.01 & $1.01 \pm 0.06$ & $0.75-1.24$ & $1.00 \pm 0.05$ & $0.83-1.17$ \\
\hline & SNP & 0.93 & 0.88 & 0.05 & $1.02 \pm 0.21$ & $0.29-1.73$ & $1.00 \pm 0.18$ & $0.58-1.83$ \\
\hline & FGN & 0.96 & 0.86 & 0.10 & $1.00 \pm 0.24$ & $0.20-1.82$ & $1.02 \pm 0.22$ & $0.53-1.90$ \\
\hline & $\mathrm{SF}$ & 1.03 & 0.99 & 0.04 & $1.00 \pm 0.12$ & $0.12 \pm 1.61$ & $1.02 \pm 0.12$ & $0.70-1.75$ \\
\hline & TGW & 0.98 & 1.05 & -0.07 & $0.92 \pm 0.16$ & $0.37 \pm 1.64$ & $1.011 \pm 0.07$ & $0.75-1.34$ \\
\hline & GL & 1.05 & 1.04 & 0.00 & $1.01 \pm 0.05$ & $0.78-1.37$ & $1.00 \pm 04$ & $0.82-1.45$ \\
\hline & GW & 0.98 & 0.98 & 0.00 & $1.01 \pm 0.05$ & $0.67-1.38$ & $1.00 \pm 04$ & $0.78-1.19$ \\
\hline & GLW & 1.09 & 1.06 & 0.03 & $1.00 \pm 0.08$ & $0.53-1.44$ & $1.01 \pm 0.06$ & $0.75-1.86$ \\
\hline
\end{tabular}


TABLE 3. Elite lines that were tolerant to low temperature, drought and bacteria blight tolerances.

\begin{tabular}{|c|c|c|c|c|c|c|c|c|c|}
\hline \multicolumn{4}{|c|}{ BB } & \multicolumn{4}{|c|}{ CT } & \multicolumn{2}{|c|}{ DT } \\
\hline DQ_3 & DQ_103 & DQ_207 & DQ_312 & DQ_149 & DQ_180 & DQ_212 & DQ_272 & DQ_15 & DQ_237 \\
\hline DQ_6 & DQ_108 & DQ_208 & DQ_315 & DQ_150 & DQ_181 & DQ_213 & DQ_283 & DQ_34 & DQ_238 \\
\hline DQ_7 & DQ_122 & DQ_214 & DQ_328 & DQ_151 & DQ_182 & DQ_214 & DQ_301 & DQ_68 & DQ_239 \\
\hline DQ_14 & DQ_135 & DQ_215 & DQ_329 & DQ_155 & DQ_183 & DQ_215 & DQ_302 & DQ_82 & DQ_244 \\
\hline DQ_17 & DQ_137 & DQ_218 & DQ_334 & DQ_156 & DQ_184 & DQ_216 & DQ_326 & DQ_146 & DQ_253 \\
\hline DQ_19 & DQ_138 & DQ_219 & DQ_339 & DQ_159 & DQ_185 & DQ_217 & DQ_333 & DQ_212 & DQ_274 \\
\hline DQ_23 & DQ_139 & DQ_221 & DQ_344 & DQ_160 & DQ_186 & DQ_218 & DQ_341 & DQ_214 & DQ_288 \\
\hline DQ_27 & DQ_140 & DQ_224 & DQ_347 & DQ_162 & DQ_187 & DQ_219 & DQ_349 & DQ_215 & DQ_291 \\
\hline DQ_60 & DQ_141 & DQ_227 & DQ_373 & DQ_163 & DQ_188 & DQ_222 & DQ_388 & DQ_219 & DQ_309 \\
\hline DQ_64 & DQ_144 & DQ_230 & DQ_435 & DQ_164 & DQ_190 & DQ_223 & & DQ_225 & DQ_372 \\
\hline DQ_65 & DQ_145 & DQ_231 & DQ_462 & DQ_165 & DQ_194 & DQ_224 & & DQ_227 & DQ_384 \\
\hline DQ_82 & DQ_148 & DQ_232 & DQ_464 & DQ_167 & DQ_195 & DQ_226 & & DQ_230 & DQ_385 \\
\hline DQ_90 & DQ_153 & DQ_242 & DQ_474 & DQ_168 & DQ_196 & DQ_227 & & DQ_231 & DQ_386 \\
\hline DQ_92 & DQ_159 & DQ_248 & & DQ_169 & DQ_197 & DQ_228 & & DQ_232 & DQ_389 \\
\hline DQ_93 & DQ_167 & DQ_250 & & DQ_170 & DQ_198 & DQ_229 & & DQ_237 & DQ_390 \\
\hline DQ_94 & DQ_176 & DQ_271 & & DQ_172 & DQ_199 & DQ_236 & & DQ_288 & DQ_393 \\
\hline DQ_95 & DQ_184 & DQ_281 & & DQ_175 & D Q_201 & DQ_238 & & DQ_386 & DQ_415 \\
\hline DQ_96 & DQ_194 & DQ_288 & & DQ_176 & DQ_205 & DQ_260 & & DQ_389 & DQ_461 \\
\hline DQ_99 & DQ_196 & DQ_291 & & DQ_177 & DQ_206 & DQ_266 & & DQ_390 & \\
\hline DQ_100 & DQ_198 & DQ_310 & & DQ_178 & DQ_207 & DQ_267 & & DQ_393 & \\
\hline DQ_101 & DQ_203 & DQ_311 & & DQ_179 & DQ_208 & DQ_271 & & DQ_461 & \\
\hline
\end{tabular}

Red $=$ Bacterial blight and Drought tolerant. Blue $=$ Bacterial blight and low-temperature tolerant. $\mathrm{CT}$, Purple $=$ Low-temperature and drought tolerance, Green $=$ bacterial blight, low-temperature and drought tolerances simultaneously.

containing QTLs controlling multiple stresses tolerance were from japonica background (parent B), and matched with phenotyping data in Table 1 that shows lines from japonica background were more tolerant to low temperature than lines from indica background.

QTL analysis detected multiple drought tolerance QTLs between the markers; M24M26 (Ch1), M36-M37 and M53-M54 (ch2), M111-M112 (ch4), M117-M118 and M130M131 (ch5), M137-M139 (ch6), M180-M181 (ch8) and M219-M220 (ch10). These regions concur with the introgression segments of chromosomes of the lines resistant to multiple stresses. These results indicate that overlapping regions can be inherited and expressed on natural environment. Thus proper phenotyping and genotyping together can lead to the development of rice variety resistant to multiple stresses.

\section{DISCUSSION}

Low temperature, drought and bacterial blight stress tolerance lines. This study has revealed occurrence of a number of transgressive lines, with trait values significantly higher than the recurrent parents under all stresses (Table 1), providing a platform for developing a rice variety with improved grain yield under adverse environments; suggesting plants respond to various stresses in a similar way. The correlation coefficient showed that grain shape traits were the least affected by stresses, GNP and FGN moderately affected; while GY ( $r=0.27$ was most affected.

Overlap of drought tolerance, BB resistance and low-temperature QTLs. Figure 1 shows chromosome segments involved in controlling yield and yield related 
Legend

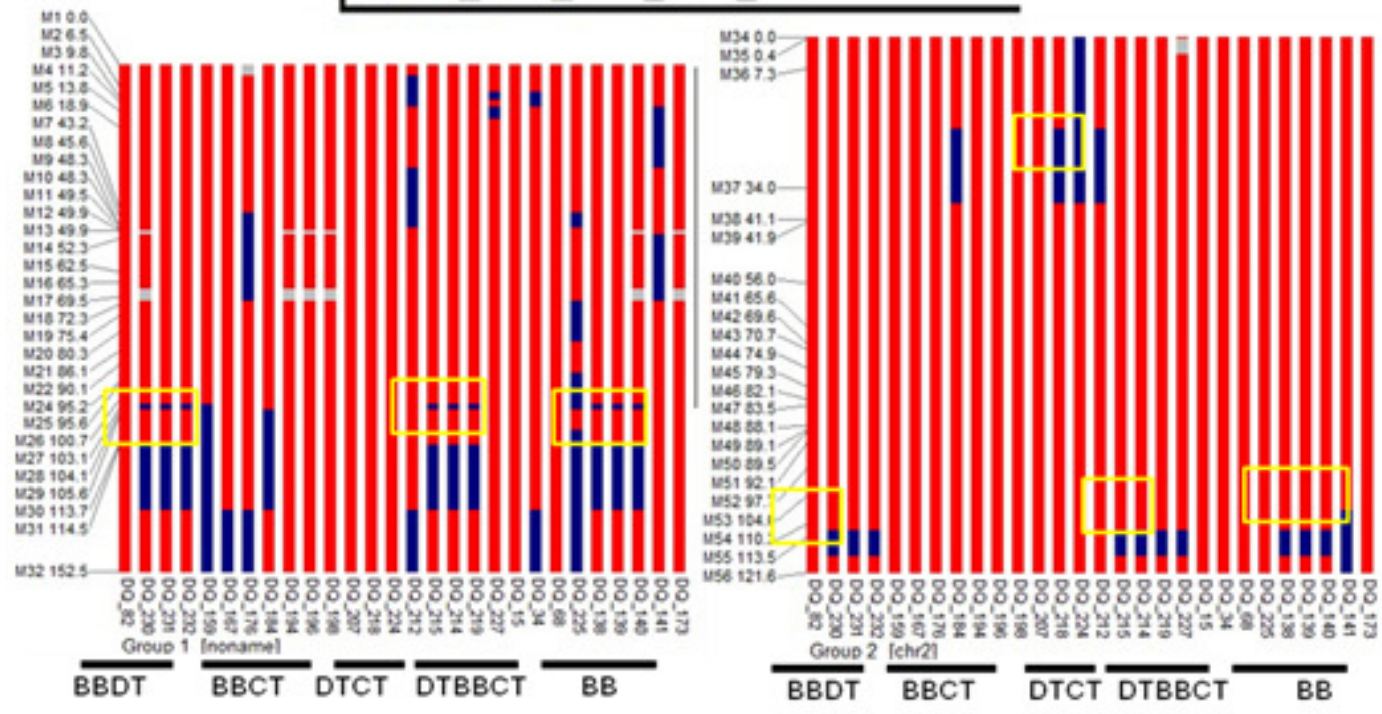

BB DT: $82,230,231,232, B B ;$ CT: 159,167, 176, 184, 194, 196, 198,217, 218, 224 DTCT: 212; DTBBCT:214,215, 219227.

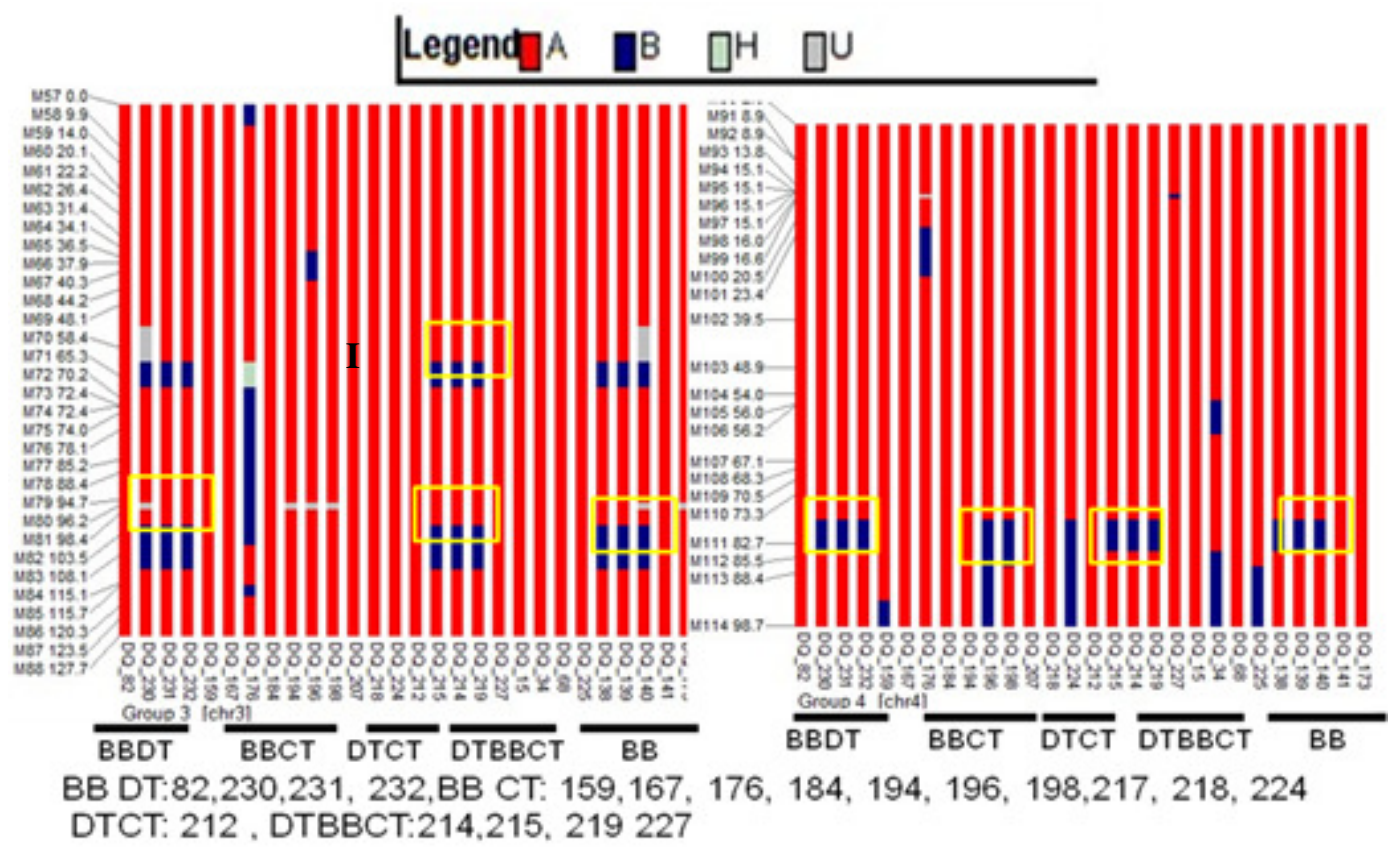

Figure 1. Introgression patterns of the elite rice lines resistant to multiple stresses in MH64_IL background. BB bacterial blight, CT low temperature tolerant, DT drought tolerant. 


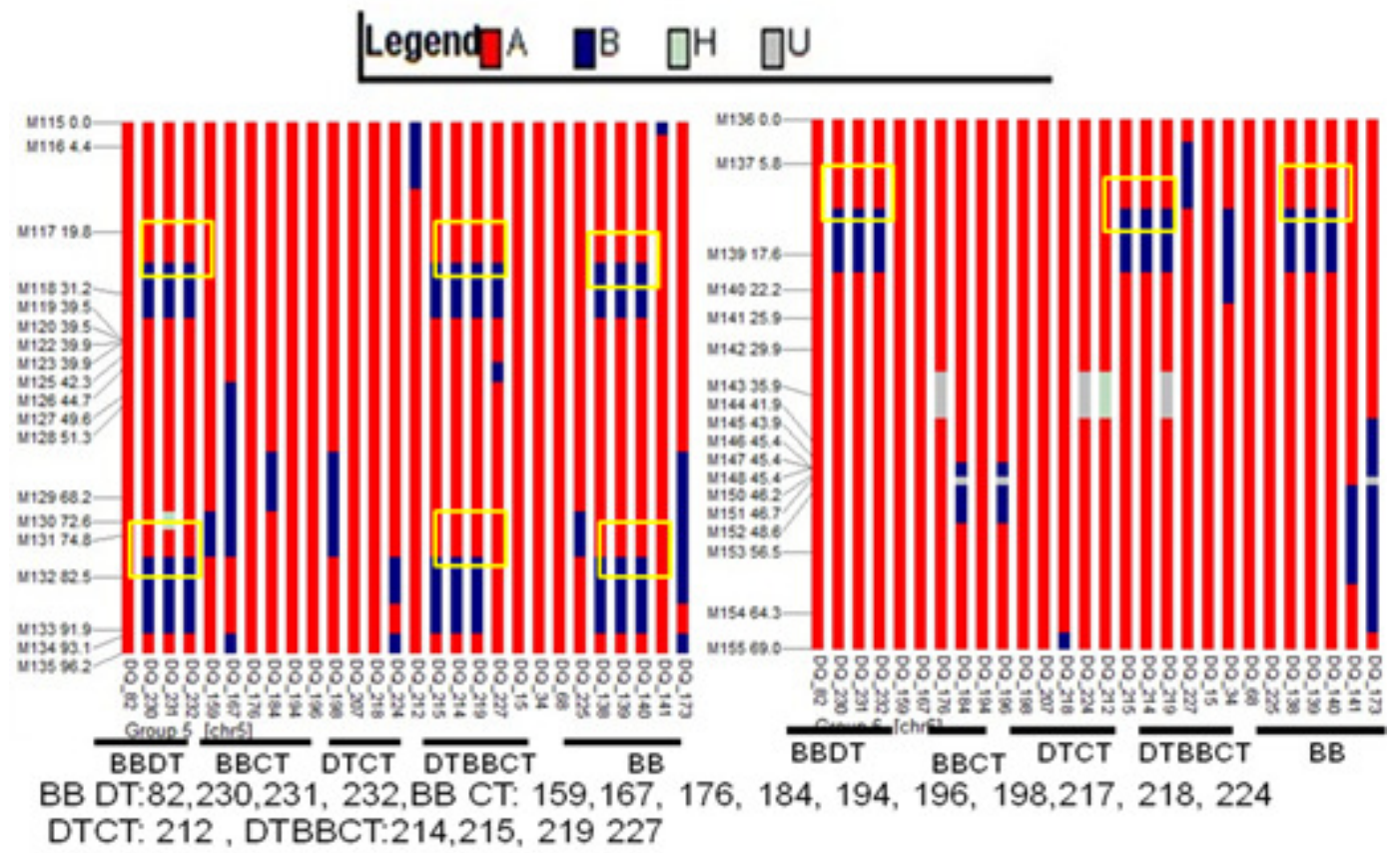

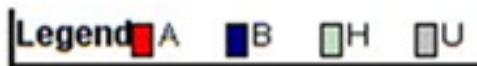

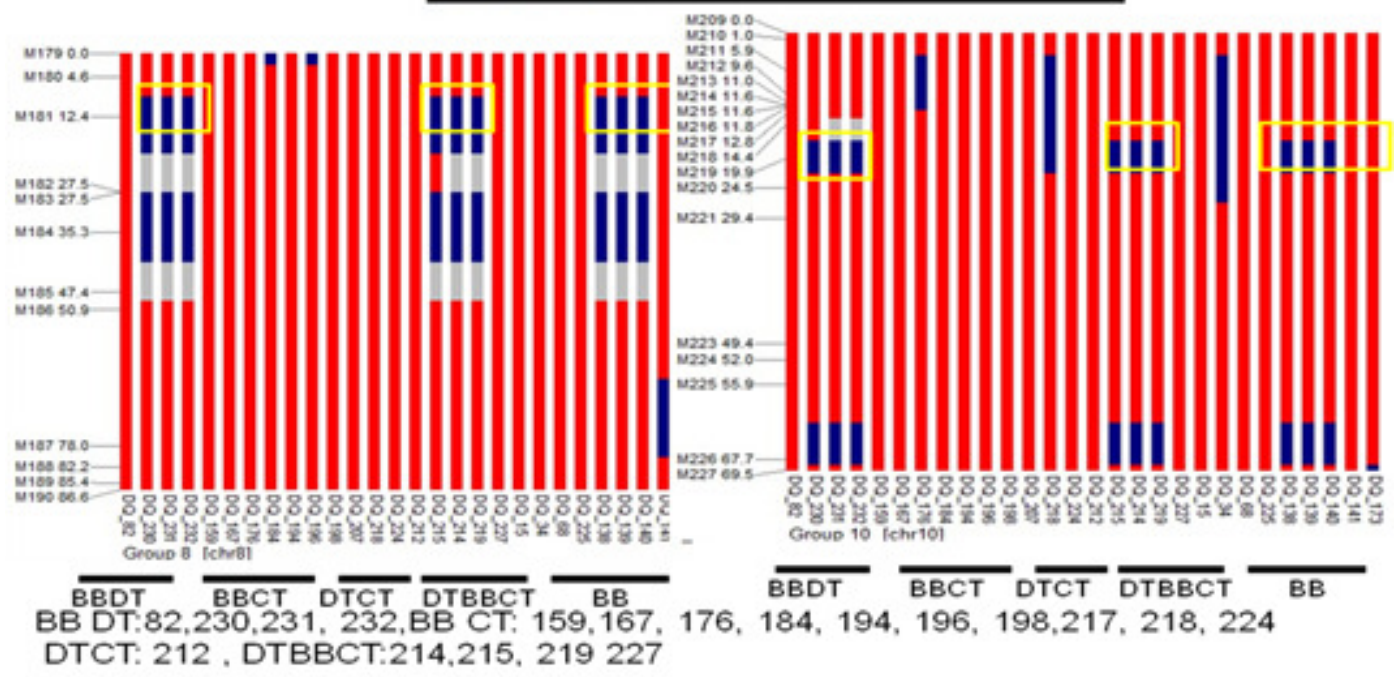

Figure 2. Introgression patterns of the elite rice lines resistant to multiple stresses in MH63 background. BB bacterial blight, CT low temperature tolerant, DT drought tolerant continues.

traits under drought stress, BB stress and lowtemperature stresses. In chromosome 1, there were three overlapping regions; the first region was located between M5-M6. This region harboured qsnland $q f g l$ responsible for spikelet number and filled grain number which was identified under drought stress, BB stress as well as under normal conditions in 02428_IL populations and MH63_IL populations, respectively. The second region 
in chromosome 1 was located between M19M27. This region harboured two QTLs, qph1 and qpll responsible for plant height and panicle length. These QTLs were also genetic background dependent, common in drought, $\mathrm{BB}$ and normal irrigation conditions in MH63_BILs backgrounds only.

Chromosome 2 had four regions containing overlapping QTLs. The first region flanked with markers M37-M38 had HD for days to heading detected under drought, BB and normal conditions (DBN) and $\mathrm{PN}$ responsible for panicle number detected under BB and lowtemperature only. These QTLs were also genetic background dependant. Adjacent to this region was M40-M44, where qsn4 for total spikelet number was detected under DBN conditions in 02428_BILs population.

The same QTL was found in MH63_IL population under DS. In this region salt tolerance QTL has been reported (Wang et al., 2012). The third region located between markers M48-49M had $q g l 2$ for grain length, $q g w 2$ for grain width under DBN conditions were detected. The fourth region lied between M54-M55 harboring qgy2 for grain yield per plant under DB conditions (Fig. 3). In chromosome 3, two major regions one located between markers M71-M78 and the second region located between M82-M83 harbouring QTLs controlling yield related traits, were detected. The first regions had three QTLs $q p l 3$ and $q g l 3$ for panicle and grain length, respectively; both detected under DBN conditions in MH63_IL and 02428_IL populations.

Under the same region and conditions, $q p h 3$ for plant height was also detected in 02428_IL population. In this region, days to seedling survival in salt stress have also been reported (Wang et al., 2012). The second region had $q f g 3, q s n 3$ and $q p n 3$ for filled grain number, spikelet number per panicle and panicle number between M82-M83 but in MH63_IL populations only in DCBN conditions.

In chromosome 4, there were two main QTL overlapping regions; the first region was located between M105-M106 harbouring qhd4 for heading date detected under DCN conditions in MH63_BILs and the second region was located between, M110-M112 harbouring $q p n 4$ and $q f g 4$ in both populations under DCBN conditions. On the same region and similar conditions, qgl4 for grain length was detected in MH63_IL population.

In chromosome 5, there were three regions containing overlapping QTLs. The first region was located between M117-M118 harbouring $q p h 5$ for plant height, $q s n 5$ for spikelet number and $q g w 4$ for grain width detected under DBN. In this region, $x a 5$ which is a recessive gene located at $46.2 \mathrm{cM}$, also contained $q c 5, q v 5$ responsible for BB stress resistance has been cloned. Thus, this is a hotspot region that can be utilised to combine BB resistance, DS tolerance and high yield potential. The second region in chromosome 5 had $9 g 15$ for grain length detected under DB in 02428_IL, and $q p n 5$ for panicle number detected under DBN conditions in MH63_IL background only. The third region between M132-M134 contained qtw5 for 1000-grain weight detected under DBN in 02428_IL populations only.

In chromosome 6 there were 3 regions containing overlapping QTLs. The first region lied between M137-M138 harboring qpn6 for panicle number, $q p h 6$ for plant height and $q p l 6$ for panicle length all in MH63_IL population only detected under DBN conditions. The second region M142-M143 had qgw6 for grain width detected in 02428_IL populations under DBN conditions. The third region M153-M154 had qgy6 for grain yield per plant. This QTL was detected under DBN conditions in MH63_IL populations only.

In chromosome 7 there were also three regions harboring overlapping QTLs. The first region was located between M158-M159 had $q p h 7$ for plant height, and lastly M177-M178 harboring $q p n 7$ for panicle number. QTLs $q p h 7$ and $q p n 7$ detected conditions (CBN). Between M168-M169, qgy 7 for grain yield per plant was detected under DBN conditions in MH63_IL populations only.

In chromosome 8, overlapping QTLs were located between M179-M180 harbouring $q f g 8$ 

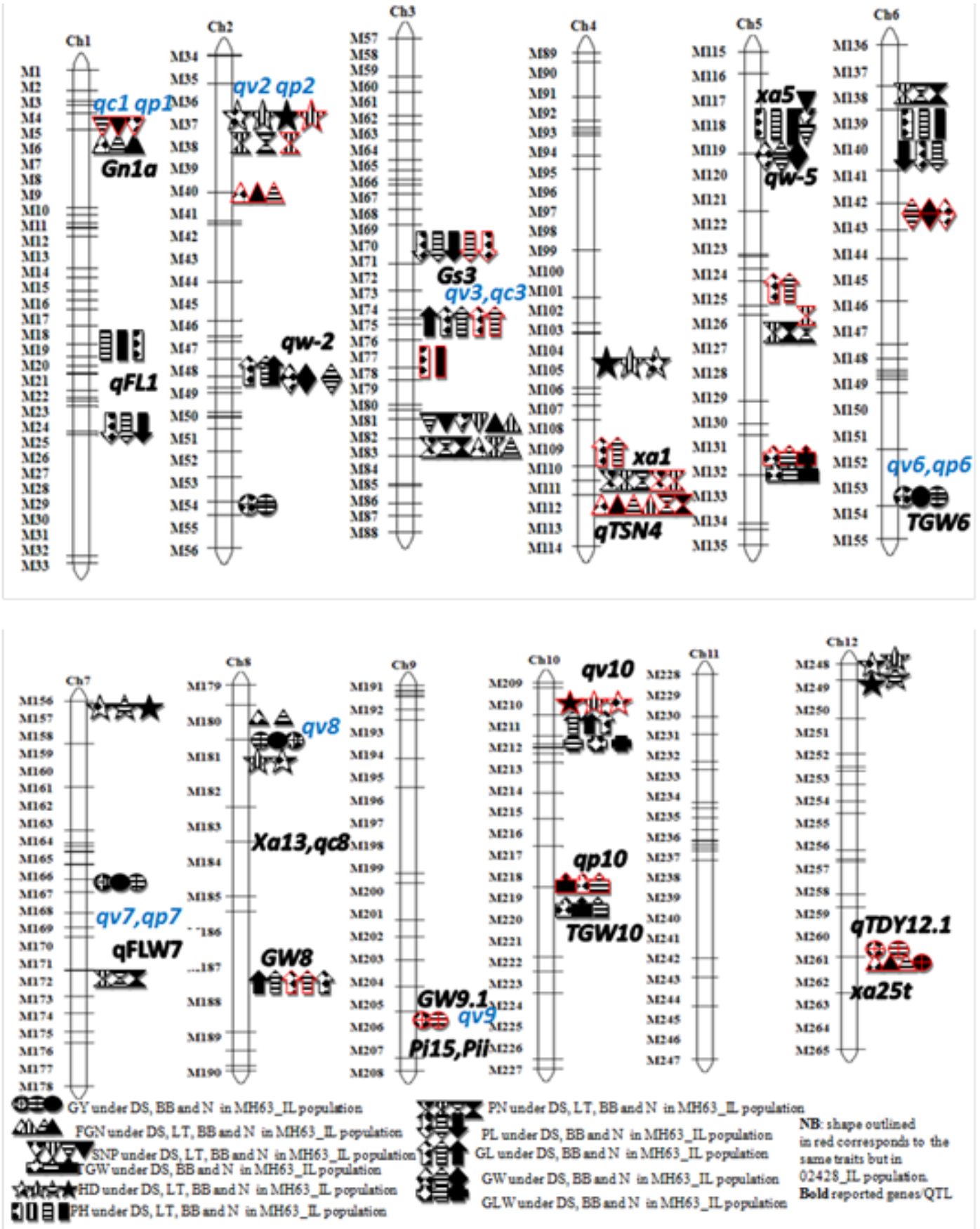

Figure 3. Quantitative trait loci illustrating genetic overlapping regions for the QTLs affecting drought, BB and low-temperature tolerances in rice. $\mathrm{Ch}$,chromosome. 
for filled grain number in DB stresses, qgy 8 for grain yield, and $q h d 8$ for heading date between M183-M184 were detected under DBN and DC stresses, respectively, in MH63_IL background. A major BB resistant QTL to $\mathrm{BB}$ race $\mathrm{C} 5$ and race $\mathrm{V} 5$ was also detected in this region. Therefore, it is an important region for yield improvement under DBN environments. Adjacent to this region between M188-M189, a genetic background independent QTL, qgl8 responsible for grain length detected under DBN conditions in both MH63_IL and 02428_IL populations, was detected.

In chromosome 9 , in the region between M206-M209, there was qgy9 under DB environments detected in 02428_IL populations only. In chromosome 10, there were two regions harbouring overlapping QTLs. The first region, located between M212-M214, had qph10 for heading date detected under DCN environments in 02428_IL populations, ql10 for grain length and $q g l w 8$ for grain length width ratio both under DBN environments in MH63_Il populations only. The second region in the chromosome 10 was located between M221-M222. This region harboured qtw10 controlling 1000-grain weight detected under DBN conditions in both populations. This QTL is synonymous to $T G W_{10}$ responsible for high grain weight identified from Azucena $\times$ IR64 population (Singh et al., 2015). Chromosome 11 as indicated in Figure 3 had overlapping QTLs. In chromosome 12, between markers M249-M250, there was qhd12 for heading date in DCBN conditions in MH63_IL populations. Other overlapping QTLs were detected between M262-M264 harboring qgy 12 and $q f g 12$ for grain yield, and field grain number, respectively. These QTLs were detected under DBN conditions in 02428_IL populations only. In this region, a major QTL for upland drought tolerance $q D T Y 12.1$, in the Vandana/Way Rarem population with pleiotropic effect on (FGN), panicle number and 1000-grain weight (TGW) have been reported (Bernier et al., 2007). Up to date, rice response to a combination of both low temperature and drought, $\mathrm{BB}$ and drought or all $\mathrm{BB}$, drought and low-temperatures in rice have not been reported. However, lowtemperature and drought in soybeans (Zhang et al., 2012) salt and low temperature in soybeans (Peng et al., 2011), drought and sheath blast in rice (Zheng et al., 2007) salt and drought in rice (Xu et al., 2011; Wang et al., 2012) have been reported. Genetic relationships and molecular mechanisms underlying $\mathrm{BB}$, DS and CT reported in this study provides a basic information to understand interrelationships of these biotic and biotic stresses in field conditions.

\section{ACKNOWLEDGEMENT}

I acknowledge Chinese Academy of Agricultural Sciences for hosting my research and the Chinese Scholarship Council for providing funding.

\section{REFERENCES}

Abuqamar, S., Luo, H., Laluk, K., Mickelbart, M.V. and Mengiste, T. 2009. Crosstalk between bioticand abiotic stress responses in tomato is mediated by the AIM1 transcriptionfactor. Plant Journal 58:34760.

Bernier, J., Kumar, A., Ramaiah, V., Spaner, D. and Atlin, G. N. 2007. A large-effect QTL for grain yield under reproductive-stage drought stress in upland rice. Crop Science 47: 507-516.

Cao, F.Y., Yoshioka, K. and Desveaux, D. 2011 The roles of ABA in plant pathogen interactions. Journal of Plant Research 2(124):489-499.

Epple, P., Mack, A.A., Morris,V.R.F. and Dang, J.L. 2003. Antagonistic control of oxidative stress-induced cell death in Arabidopsis by two related, plant specific zinc finger proteins. Proceedings of the National Academy of Science, USA 100:6831-6836 
Kauffman, H.E., Reddy, A., Hsieh, S.P.Y. and Merca, S.D. 1973. An improved technique for evaluating resistance of varieties to Xanthomonas oryzae pv. oryzae. Plant Disease. Reporter 57:537-541.

Liu, R.H and Meng, J.L. 2003. MapDraw: A Microsoft Excel macro for drawing genetic linkage maps based on given genetic linkage data. Heraditas 25:317-321.

Ou, S.H. 1985. Rice diseases, 2nd edn. Kew, UK: Commonwealth Mycological Institute, $379 \mathrm{pp}$.

Peng-Cheng, Q., Wen-Bo, Z., Hong-Wei, J., Chun-Yan, L., Can-Dong, L., Dong-Mei, F. and Qing-Shan, C. 2011. Genetic overlap between salt and low-temperature tolerance loci at germination stage of soybean. Scientia Agricultura Sinica 44: 1980-1988.

Ralph, V.B. 1999. GGT graphical genotyping software user manual - 2007 Edition.

Ramegowda, V. and Senthil-Kumar, M. 2015. The interactive effects of simultaneous biotic and abiotic stresses on plants: Mechanistic understanding from drought and pathogen combination. Journal of Plant Physiology 176: 47-54.

Shaik, R. and Ramakrishna, W. 2014. Machine learning approaches distinguish multiple stress conditions using stress-responsive genes and identify candidate genes for broad resistance in rice. Plant Physiology164: 481-495.

Sharma, R., Vleesschauwera, D.D., Sharmaa, M. and KRonalda, P.C. 2013. Recent advances in dissecting stress-regulatory crosstalk in rice. Molecular Plant 6:250260.

Singh, N., Shahana, M., Singh, O.N., Prashant, V., Singh, A.K. and Sanjay, S.
2015. A large-effect QTL for grain weight in rice on chromosome 10. Australian Journal of Crop Science 9:372-377

Wang, Y., Zang, J., Sun, Y., Ali, J., Xu, J. and $\mathrm{Li}, \mathrm{Z}$. 2012. Identification of genetic overlaps for salt and drought tolerance using simple sequence repeat markers on an advanced backcross population in rice. Crop Science 52:1583-159.

Wang, J., Li, H., Zhang, L. and Meng, L. 2016. Users' Manual of QTL IciMapping Version 4.0. The quantitative genetics group, Institute of Crop Science, Chinese Academy of Agricultural Sciences (CAAS), Beijing 100081, China, and Genetic Resources Program, International Maize and Wheat Improvement Center (CIMMYT),. Apdo. Postal 6-641, 06600 Mexico, D.F., Mexico.

Xu, G.Y., Rocha, P.S., Wang, M.L., Xu, Y.C., Cui, L.Y. and Li, Y.X. 2011. A novel rice calmodulin-like gene, OsMSR2, enhances drought and salt tolerance and increases ABA sensitivity in Arabidopsis. Planta 234:47-59.

Zhang, W.B., Qiu, P. C., Jiang, H.W., Liu, C.Y., Xin, D.W., Li, C.D. and Chen, Q.S. 2012. Dissection of genetic overlap of drought and low-temperature tolerance QTLs at the germination stage using backcross introgression lines in soybean. Molecular Biology Reports 39:6087-6094.

Zheng, T.Q., Xu, J.L., Fu, B.Y., Gao, Y.M., Satish, V., Renee, L., Zhai, H.Q., Wan, J.M., Zhu, L.H. and LI, Z.K. 2007. Preliminary identification of genetic overlaps between sheath blight resistance and drought tolerance in the introgression lines from directional selection. Acta Agronomy Sinica 33:1380-1384. 